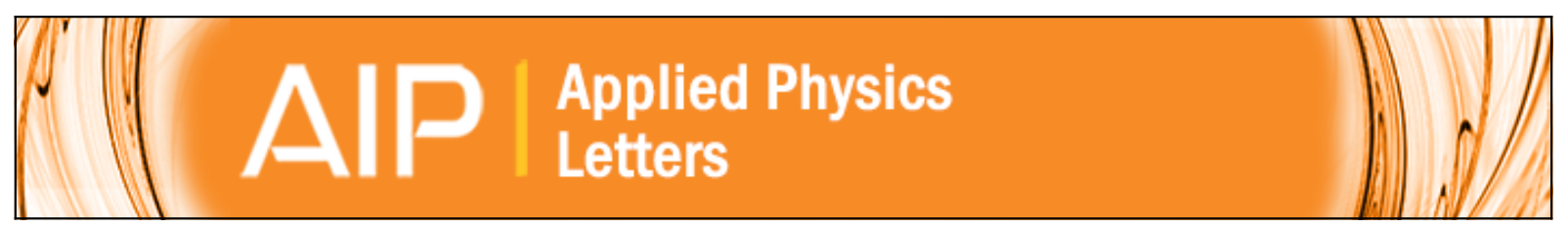

\title{
On-the-fly scans for X-ray ptychography
}

Philipp M. Pelz, Manuel Guizar-Sicairos, Pierre Thibault, Ian Johnson, Mirko Holler, and Andreas Menzel

Citation: Applied Physics Letters 105, 251101 (2014); doi: 10.1063/1.4904943

View online: http://dx.doi.org/10.1063/1.4904943

View Table of Contents: http://scitation.aip.org/content/aip/journal/apl/105/25?ver=pdfcov

Published by the AIP Publishing

\section{Articles you may be interested in}

Efficient use of coherent X-rays in ptychography: Towards high-resolution and high-throughput observation of weak-phase objects

Appl. Phys. Lett. 108, 071103 (2016); 10.1063/1.4942105

Nanofabrication of custom X-ray optical components

AIP Conf. Proc. 507, 704 (2000); 10.1063/1.1291236

Compound refractive lenses for X-ray microanalysis

AIP Conf. Proc. 507, 694 (2000); 10.1063/1.1291234

X-ray microbeams from waveguide optics

AIP Conf. Proc. 507, 631 (2000); 10.1063/1.1291222

Current status of the Scanning X-ray Microscope at the ESRF

AIP Conf. Proc. 507, 458 (2000); 10.1063/1.1291190

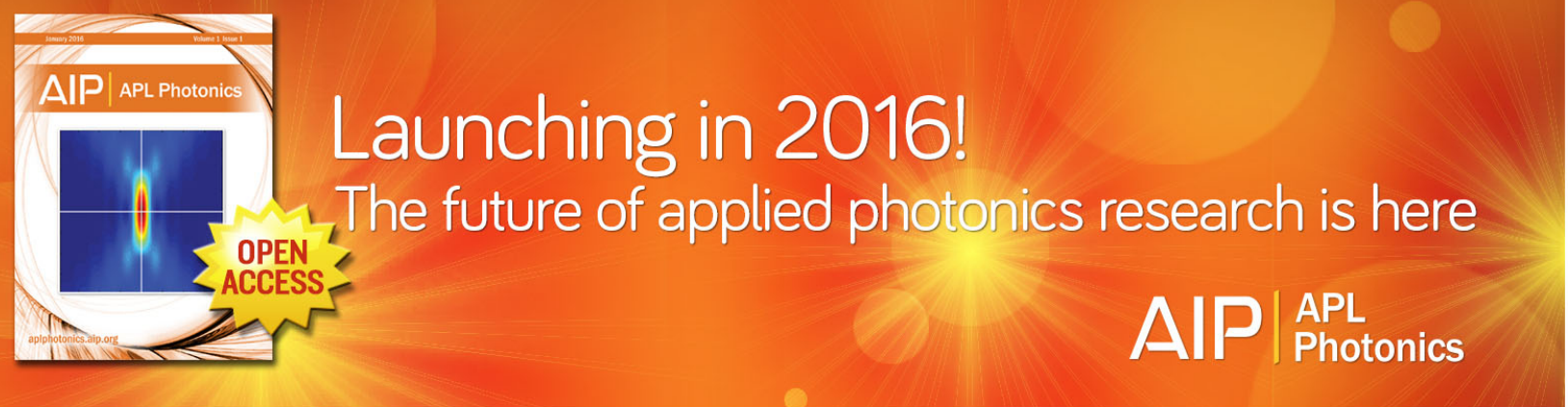




\title{
On-the-fly scans for X-ray ptychography
}

\author{
Philipp M. Pelz, ${ }^{1,2,3,4, a)}$ Manuel Guizar-Sicairos, ${ }^{1}$ Pierre Thibault, ${ }^{5}$ Ian Johnson, ${ }^{1, b)}$ \\ Mirko Holler, ${ }^{1}$ and Andreas Menzel ${ }^{1}$ \\ ${ }^{1}$ Paul Scherrer Institut, 5232 Villigen PSI, Switzerland \\ ${ }^{2}$ Lehrstuhl für Biomedizinische Physik, Physik-Department, Technische Universität München, \\ 85747 Garching, Germany \\ ${ }^{3}$ University of Montpellier 2, UMR 5253, ICGM, C2M, CC1504, Montpellier, France \\ ${ }^{4}$ Dipartimento di Chimica Inorganica, Chimica Fisica e Chimica dei Materiali, Universita di Torino, I-10125, \\ Torino, Italy \\ ${ }^{5}$ Department of Physics and Astronomy, University College London, London, United Kingdom
}

(Received 5 October 2014; accepted 11 December 2014; published online 22 December 2014)

\begin{abstract}
With the increasing importance of nanotechnology, the need for reliable real-time imaging of mesoscopic objects with nanometer resolution is rising. For X-ray ptychography, a scanning microscopy technique that provides nanometric resolution on extended fields of view, and the settling time of the scanning system is one of the bottlenecks for fast imaging. Here, we demonstrate that ptychographic on-the-fly scans, i.e., collecting diffraction patterns while the sample is scanned with constant velocity, can be modelled as a state mixture of the probing radiation and allow for reliable image recovery. Characteristics of the probe modes are discussed for various scan parameters, and the application to significantly reducing the scanning time is considered. (C) 2014 AIP Publishing LLC. [http://dx.doi.org/10.1063/1.4904943]
\end{abstract}

$\mathrm{X}$-ray ptychography is a lensless imaging method that produces high-resolution maps of the complex transmission function of extended samples by combining multiple coherent diffraction measurements from the illumination of several overlapping regions on the specimen. ${ }^{1-3}$ It holds great promise as a microscopy tool for $2 \mathrm{D}$ and $3 \mathrm{D}$ analysis at the nanoscale with applications in materials science ${ }^{4,5}$ and biology. ${ }^{6,7}$ However, its nature as a scanning technique poses strict requirements on the instrumentation. Recent advances have helped to alleviate many problems, including positioning errors ${ }^{8,9}$ and stability ${ }^{10}$ as well as partial-coherence effects and fluctuations. ${ }^{11,12}$ For fast measurements that require a small exposure time, the overhead caused by detector readout and settling of piezoelectric scanning systems can be significant and limit the imaging throughput. On-the-fly measurements, for which the sample is scanned at a constant velocity while a point detector measures the transmission of the sample, have greatly reduced this problem for traditional scanning transmission X-ray microscopy. However, conventional reconstruction algorithms for ptychography inherently assume that the diffraction data are acquired on a static sample. We demonstrate that ptychographic scans of a continuously moving object can be reconstructed with high fidelity by allowing the simultaneous reconstruction of several mutually incoherent modes of the probing illumination.

A conventional ptychographic data set consists of a series of far-field diffraction measurements in which the sample is scanned at adjacent positions such that there is a spatial overlap of successive exposures. The measured far-

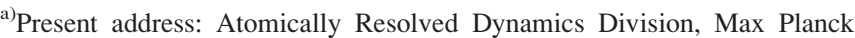
Institute for the Structure and Dynamics of Matter, Luruper Chaussee 149, 22761 Hamburg, Germany. Electronic mail: philipp.pelz@mpsd.mpg.de.

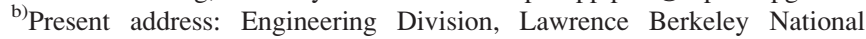
Laboratory, 1 Cyclotron Rd., Berkeley, California 94720, USA.
}

field intensity is related to the complex exit surface wave (ESW) via

$$
I_{j \vec{q}}=\left|\mathcal{F}\left[\psi_{j \vec{r}}\right]\right|^{2}
$$

where $j$ is the index of the current diffraction pattern, $\vec{q}$ is the two dimensional wave-vector in the measurement plane, and $\mathcal{F}[\cdot]$ is the two-dimensional Fourier transform. Within the projection approximation, the ESW can be expressed as a product of probe and object functions ${ }^{2}$

$$
\psi_{j \vec{r}}=P\left(\vec{r}-\vec{r}_{j}\right) O(\vec{r})
$$

For partially coherent wavefields, this model can be extended in the following way:

$$
I_{j \vec{q}}=\iint J\left(\vec{r}_{1}-\vec{r}_{j}, \vec{r}_{2}-\vec{r}_{j}\right) \rho\left(\vec{r}_{1}, \vec{r}_{2}\right) e^{i \vec{q}\left(\vec{r}_{1}-\vec{r}_{2}\right)} d \vec{r}_{1} d \vec{r}_{2},
$$

where $J\left(\vec{r}_{1}, \vec{r}_{2}\right)$ is the mutual optical intensity and $\rho\left(\vec{r}_{1}, \vec{r}_{2}\right)$ is the density function, which can both be written as ${ }^{13,14}$

$$
\begin{gathered}
J\left(\vec{r}_{1}, \vec{r}_{2}\right)=\sum_{n=1}^{N} \eta_{n} P_{n}\left(\vec{r}_{1}\right) P_{n}^{*}\left(\vec{r}_{2}\right), \\
\rho\left(\vec{r}_{1}, \vec{r}_{2}\right)=\sum_{m=1}^{M} \mu_{m} O_{m}\left(\vec{r}_{1}\right) O_{m}^{*}\left(\vec{r}_{2}\right) .
\end{gathered}
$$

$\left\{P_{n}\right\}_{n=\{1, \ldots, N\}}$ and $\left\{O_{m}\right\}_{m=\{1, \ldots, M\}}$ are the mutually incoherent, orthogonal modes of probe and object. In this case, the far-field intensity can be written as the incoherent double sum over all probe and object modes

$$
I_{j \vec{q}}=\sum_{n=1}^{N} \sum_{m=1}^{M}\left|\mathcal{F}\left[P_{n}\left(\vec{r}-\vec{r}_{j}\right) O_{m}(\vec{r})\right]\right|^{2}
$$


The symmetry of probe and object in Eq. (6) allows partial coherence effects to be accounted for by both probe and object functions. In the case of on-the-fly scans, the intensity for a coherent far-field diffraction pattern resulting from an object moving with a constant velocity over the distance $a$ along a unit vector $\hat{e}$ during exposure can be modelled as

$$
I_{j \vec{q}}=\int_{0}^{a}\left|\mathcal{F}\left[P\left(\vec{r}-\vec{r}_{j}-R \cdot \hat{e}\right) O(\vec{r})\right]\right|^{2} d R .
$$

Here, we have used the fact that in the far-field intensity distribution, there is no information on absolute positioning, so that the relative positions of probe and object can be interchanged. Numerically, the integral can be written as a sum

$$
I_{j \vec{q}}=\sum_{n=1}^{N}\left|\mathcal{F}\left[P\left(\vec{r}-\vec{r}_{j}-R_{n} \cdot \hat{e}\right) O(\vec{r})\right]\right|^{2},
$$

with a sufficiently fine sampling interval $N$ and $R_{n}=\frac{n}{N} a$. This expression can be written in the form given by Eq. (3), with $J\left(\vec{r}_{1}, \vec{r}_{2}\right)=\sum_{n=1}^{N} P_{n}\left(\vec{r}_{1}-R_{n} \cdot \hat{e}\right) P_{n}^{*}\left(\vec{r}_{2}-R_{n} \cdot \hat{e}\right)$ and $\rho\left(\vec{r}_{1}, \vec{r}_{2}\right)=O\left(\vec{r}_{1}\right) O^{*}\left(\vec{r}_{2}\right)$. The coherent probe modes are therefore given by the spectral decomposition of $J$, which is numerically equivalent to a principal component decomposition of the set of probes $\left\{P\left(\vec{r}-R_{n} \cdot \hat{e}\right)\right\}_{i=\{1, \ldots, N\}}$. This effect could also be represented by object modes, but orthogonal object modes in the Euclidean basis are frequently difficult to interpret.

Experiments were performed at the cSAXS beamline of the Swiss Light Source, Paul Scherrer Institut, Switzerland. A Fresnel zone plate (FZP) with $100 \mu \mathrm{m}$ diameter and an outermost zone of $100 \mathrm{~nm}$ was used to focus a coherent beam of $6.2 \mathrm{keV}$ X-rays. The source of size $200 \mu \mathrm{m} \times 20 \mu \mathrm{m}$ was $34 \mathrm{~m}$ upstream of the FZP and horizontally apertured to $25 \mu \mathrm{m} 22 \mathrm{~m}$ upstream of the FZP to increase transverse coherence. Longitudinal coherence was determined by the bandwidth of $0.02 \%$ of the double-crystal $\mathrm{Si}(111)$ monochromator. The sample was placed approximately $55 \mathrm{~mm}$ behind the FZP, i.e., $5 \mathrm{~mm}$ behind the focus, so that the probe incident on the object was a diverging beam of roughly $10 \mathrm{~mm}$ size. An order sorting aperture was installed close to the focus to block all but the first diffraction order of the FZP.

A single-photon counting Eiger pixel detector, ${ }^{15}$ which consisted of $514 \times 1030$ pixels of $75 \mu \mathrm{m}$ size, was placed $7.2 \mathrm{~m}$ downstream from the object. For the reconstruction, we cropped the diffraction patterns to $460 \times 460$ pixels. The detector was operated in a parallel acquire and readout mode which utilizes the on-chip buffering of the current image to achieve a $10 \mu$ s dead time between frames. The volume between sample and detector was flushed with helium to reduce absorption and scattering of the beam. The sample was a part of a computer processor ${ }^{16}$ with minimum feature size of $100 \mathrm{~nm}$.

Scanning of the sample stage in a plane perpendicular to the X-ray direction was achieved using a 2D piezoelectric translation stage (Physik Instrumente P-733.2CL) mounted on stepper motors (Newport MFA-PP). This modular test setup featured in these measurements a $200 \mathrm{~ms}$ repositioning delay. More optimized setups exist and are under development. Yet even systems with settling times in the order of $\sim 10 \mathrm{~ms}$ will be challenged by upcoming high-brightness sources. ${ }^{17}$ For on-the-fly scans, the piezo motors were programmed to scan with constant velocity along horizontal lines. A constant speed was achieved by increasing the piezo velocity to a fixed value which triggered the detector to start an acquisition series. Back to back exposures, with the negligible $10 \mu$ s of dead-time, were continuously acquired along the line scan, so that no repositioning between scan positions was necessary.

Two different step sizes between adjacent scan points were used, $250 \mathrm{~nm}$ and $1 \mu \mathrm{m}$, with line separations of $250 \mathrm{~nm}$ and $1 \mu \mathrm{m}$, respectively, which resulted in scans of $20 \times 20$ and $5 \times 5$ positions for a scan area of $5 \mu \mathrm{m} \times 5 \mu \mathrm{m}$. The data analysis made clear that a fine sampling between the lines is not necessary; therefore, $20 \times 5$ point scans were created from the measured data by choosing every 4 th line from the original scan.

In Fig. 1, we show selected diffraction patterns with the sample for (a) a static scan with $100 \mathrm{~ms}$ exposure time, (b) an on-the-fly scan with $200 \mathrm{~ms}$ exposure time, and (c) an on-the-fly scan with $2.5 \mathrm{~ms}$ exposure time. The effect of the continuous movement of the sample across $1 \mu \mathrm{m}$ can be seen as a loss of speckle contrast when comparing Figs. 1(a) and $1(\mathrm{~b})$.

The reconstructions in Figs. 2(a)-2(c) were performed using 100 iterations of the difference map algorithm ${ }^{11,18}$ and 300 iterations of likelihood optimization ${ }^{19}$ with a Poissonnoise model, a flat disc of $10 \mu \mathrm{m}$ diameter as initial illumination in the $n=1$ mode, and a random noise in the higher modes. The computational cost scales linearly with the number of modes and the number of recorded diffraction patterns. However, the problem is readily parallelized and distributed computing architectures can be profited from.

To assess the quality of the reconstructions, the resolution was calculated by Fourier shell correlation (FSC) ${ }^{10,20,21}$ of two independent reconstructions of scans with identical parameters.

Fig. 2(a) shows results of the reconstruction of a $5 \times 5$ point static scan with a step size of $1 \mu \mathrm{m}$ and $100 \mathrm{~ms}$ exposure time per point. The total scan time is $7.5 \mathrm{~s}$ with a total exposure time of $2.5 \mathrm{~s}$. The raw diffraction data are displayed in Fig. 1(a). It can be seen that up to $1 \times 10^{3}$ photons were recorded per detector pixel. The single-mode reconstruction achieves a resolution of $46 \mathrm{~nm}$, which improves slightly to $44 \mathrm{~nm}$ when using a reconstruction that includes 8 coherent modes. Since this is fairly close to the image pixel size of $41.5 \mathrm{~nm}$, this scan can be taken as a quality reference.
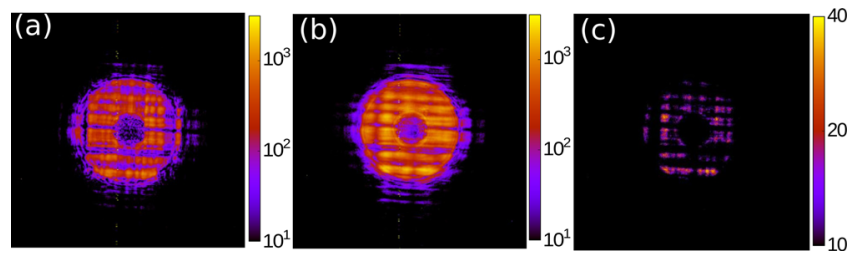

FIG. 1. Diffraction patterns (logarithmic scale) from three different scans. (a) Static scan with $5 \times 5$ points and $100 \mathrm{~ms}$ exposure time per point. (b) Onthe-fly scan with $5 \times 5$ points and $200 \mathrm{~ms}$ exposure time per point. The movement range during exposure was $1 \mu \mathrm{m}$. (c) On-the fly scan with $20 \times 5$ points and $2.5 \mathrm{~ms}$ exposure time per point. The movement range during exposure was $250 \mathrm{~nm}$. 
An on-the-fly scan with the same inter-position distance and $200 \mathrm{~ms}$ exposure time per point is shown in Fig. 2(b). The total scan time with respect to the static scan is reduced by a factor of 1.3 to $5.8 \mathrm{~s}$ due to the fewer number of repositioning delays. However, the quality of the reconstruction suffers significantly. A conventional reconstruction using a single mode, as shown in Fig. 2(b), exhibits significant blurring along the scan direction and has a resolution of $148 \mathrm{~nm}$ but still recovers overall features. A reconstruction with 12
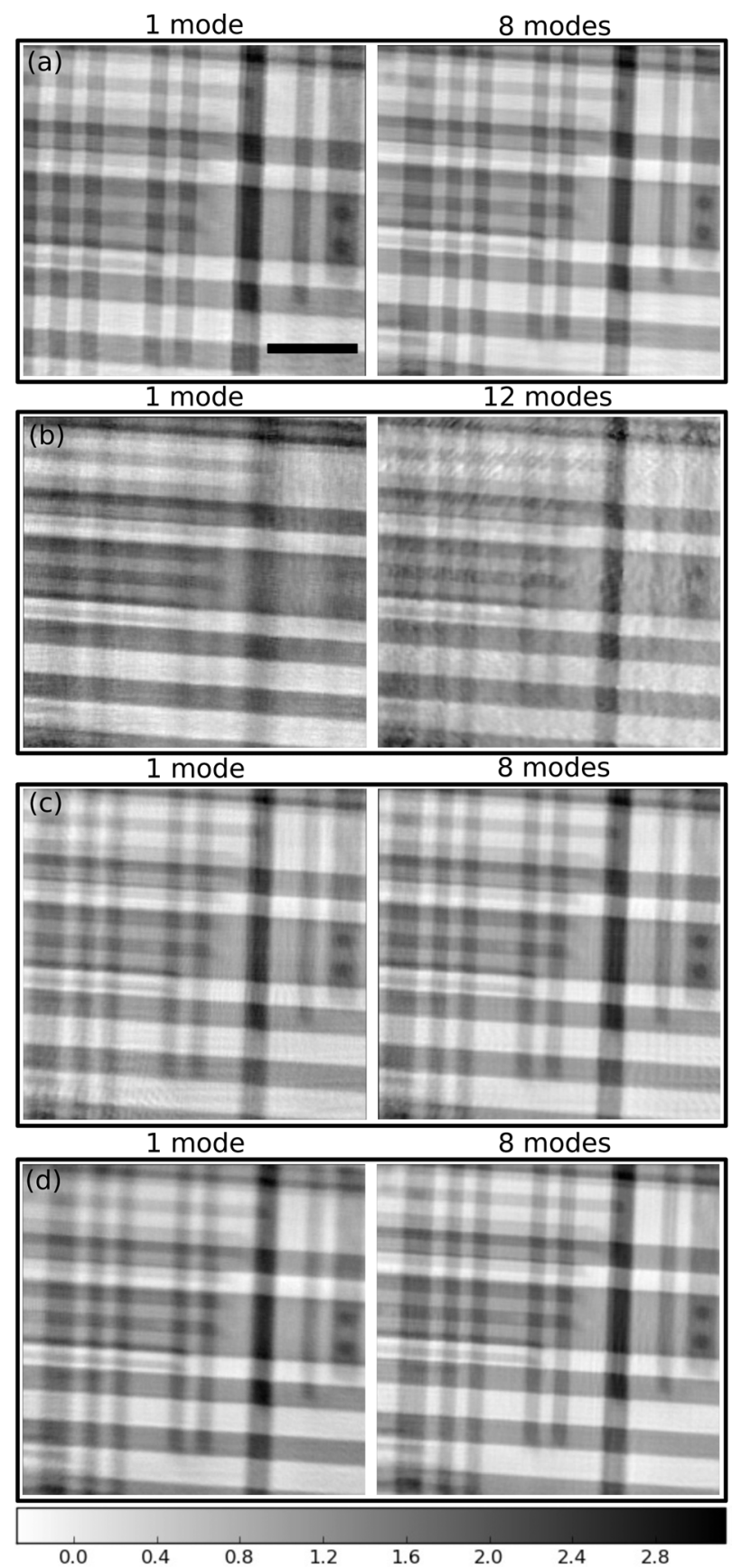

FIG. 2. Reconstructed phase of the chip sample for (a) a static scan with $5 \times 5$ points and $100 \mathrm{~ms}$ exposure time per point, (b) an on-the-fly scan with $5 \times 5$ exposures and $200 \mathrm{~ms}$ exposure time per point, (c) an on-the-fly scan with $20 \times 5$ exposures and $25 \mathrm{~ms}$ exposure time per point, and (d) an on-thefly scan with $20 \times 5$ exposures and $2.5 \mathrm{~ms}$ exposure time per point. The figures in the left column were reconstructed with one mode. The figures in the right column were reconstructed with 8 modes for (a), (c), and (d) and 12 modes for (b). The scale bar represents $2 \mu \mathrm{m}$ and the grey values are given in rad. modes shows significant improvement with $94 \mathrm{~nm}$ resolution, shown in the right panel of Fig. 2(b), but still yields relatively poor quality compared to the static scan. A decreased resolution might be the result of the population of additional modes in the illumination due to the movement, while keeping the amount of measured diffraction data constant. This is exemplified in Fig. 3, which shows reconstructions of the illumination coherent modes for various scan parameters. Notice that while for the static scan, Fig. 3(a), 90\% of the power is contained in the first two modes, for the continuous scan with $1 \mu \mathrm{m}$ step, Fig. 3(b), the power in the beam is spread out between 12 modes, and all contribute significantly to the overall beam shape with a relative power of the prominent mode of only $15.7 \%$.

Theory suggests ${ }^{11}$ that the number of measured diffraction patterns should scale linearly with the number of coherent modes that need to be reconstructed. In the case of Fig. 2(b) with only 25 diffraction patterns, there are too few data points to reconstruct all contributing modes with good quality.

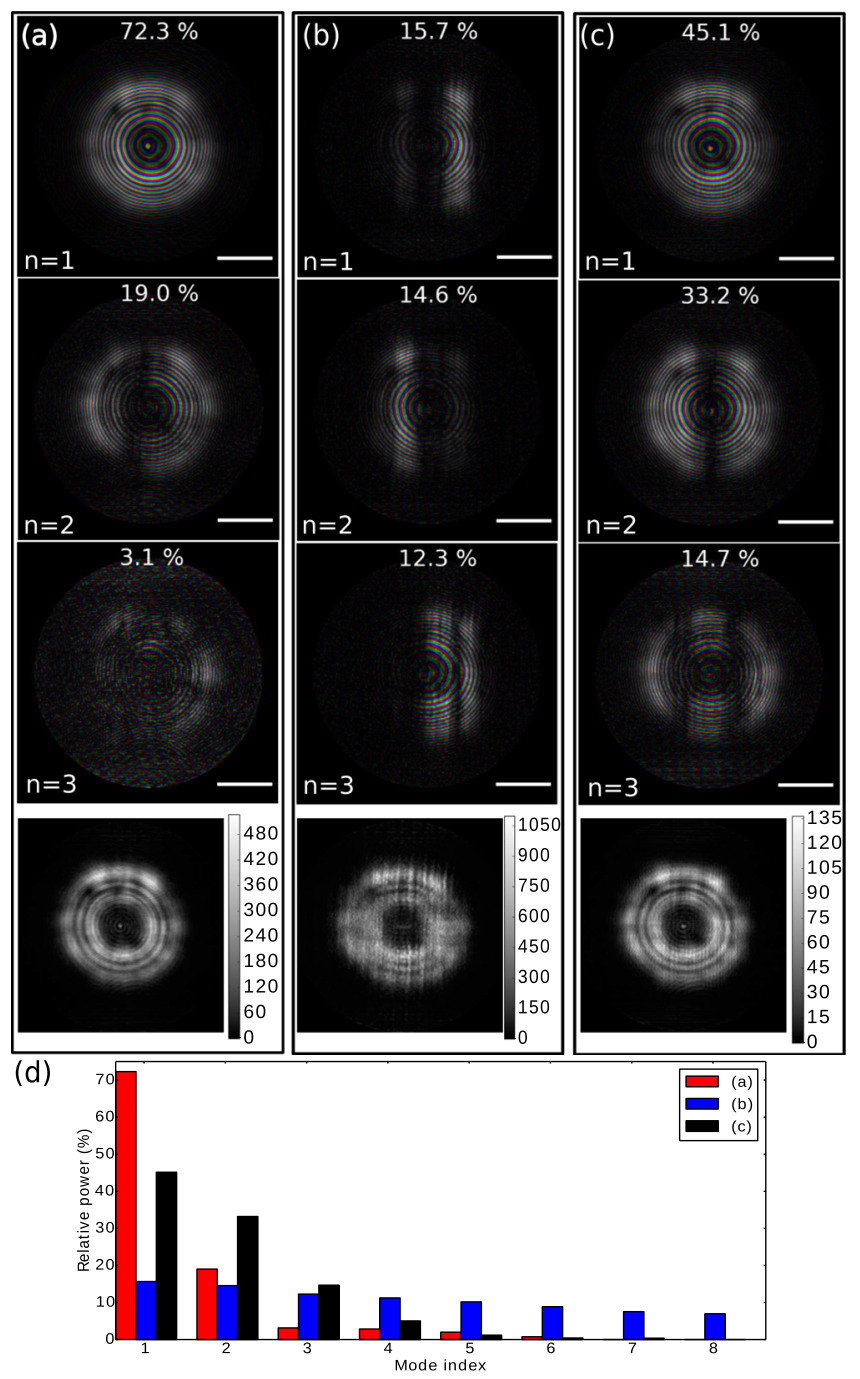

FIG. 3. The three reconstructed illumination modes with the highest relative power for (a) a $5 \times 5$ point static scan, (b) a $5 \times 5$ point on-the-fly scan, (c) a $20 \times 5$ point on-the-fly scan. Displayed at the top of each panel is the relative power of the mode. Phase and amplitude are encoded as hue and brightness, respectively, as depicted in the colorwheel. The scale bar is $4 \mu \mathrm{m}$. The bottom panel shows the beam intensity, computed from all the reconstructed modes, given in arbitrary units. (d) An overview of the relative power of the first 8 modes for the probes shown in (a)-(c) 
A remarkable improvement can be obtained by increasing the number of points along the fast scan axis, even when proportionally reducing the exposure time. In this way, we effectively reduce the relative power of the higher modes of the illumination by reducing the scan distance. This makes the reconstruction problem much more amenable by providing additional information to the algorithm through the increased number of diffraction patterns.

An example of this approach is shown in Fig. 2(c), which shows results of the reconstruction of a $20 \times 5$ point on-the-fly scan with a $250 \mathrm{~nm}$ step size, a vertical line separation of $1 \mu \mathrm{m}$, and $25 \mathrm{~ms}$ exposure time per point, leading to a total scan time of $3.3 \mathrm{~s}$ and a total exposure time of $2.5 \mathrm{~s}$. The estimated resolution is $80 \mathrm{~nm}$ for the single-mode reconstruction and $56 \mathrm{~nm}$ for the reconstruction with 8 modes. In this case, over $90 \%$ of the overall power is contained in the first three modes, as shown in Fig. 3(c). The relative power of the modes drops to 0 for $n>6$, which greatly reduces the number of variables that need to be reconstructed by the algorithm. It is therefore advisable to choose small steps along the fast axis to keep the number of significant modes low and increase the number of measured data points. The spacing along the slow axis has little effect on the reconstruction quality and need not be reduced.

On-the-fly ptychography allows a significant increase of imaging throughput by removing the inherent limitation of positioning overhead costs. To illustrate the potential speed of acquisitions of this technique, we reconstructed a $20 \times 5$ point on-the-fly scan with only $2.5 \mathrm{~ms}$ exposure time per point, leading to a total scan time of $1.05 \mathrm{~s}$ and a total exposure time of $250 \mathrm{~ms}$ where we have ignored the transfer and storage to the reconstruction workstation. In this case, the exposure time is already at a limit where the Poisson statistics in the data becomes dominant. Fig. 1(c) shows that the average photon count within the illumination diffraction cone is 5.5 photons per pixel, and noise has to be suitably accounted for in the reconstruction. For this purpose, a difference map reconstruction ${ }^{18}$ did not provide good results, and the reconstruction of this scan was performed using 300 iterations of likelihood optimization with a Poisson noise model. ${ }^{19}$ The reconstruction works well when starting from a constant disc-shaped illumination function with roughly the size of the probe for the first mode and random initialization for the higher modes. However, the convergence is aided significantly by starting from an improved initial guess for probe modes using the reconstructed illumination from a previous scan and the knowledge of the scan pattern. A good initial guess can be calculated by creating 50 shifted copies of a single-mode reconstruction of a static scan, orthogonalizing them, and taking only the $N$ most powerful modes.

The single-mode reconstruction yields an estimated resolution of $85 \mathrm{~nm}$, while a reconstruction with 8 modes yields $61 \mathrm{~nm}$ resolution, shown in Fig. 2(d), with image quality comparable to the $20 \times 5$ on-the-fly scan with $25 \mathrm{~ms}$ per point, Fig. 2(c). This indicates that resolution of the slow scan was not flux limited. Compared to the static scan, the data collection process of this on-the-fly scan is more than 7 times faster with only slightly diminished resolution. Even if the static scan was made of comparable dose with $12.5 \mathrm{~ms}$ per point, the measurement would be dominated by piezoelectric positioning and would take over $5 \mathrm{~s}$. For all onthe-fly scans presented in this paper, the exposure time overhead due to repositioning is reduced from a per-point overhead to a per-line overhead compared to conventional, static scans.

For very high resolution ptychography, ${ }^{10}$ it is much more effective to increase the number of points, effectively having more overlap between illuminated regions, than increasing the exposure time. This results in ptychography scans being often limited by piezoelectric settling overhead. The use of continuous scans allows full flexibility to trade off resolution or sensitivity for speed. Here, we demonstrate that using a mode mixture reconstruction is necessary and sufficient for high-quality reconstructions of on-the-fly ptychography and quantify the increase in scanning speed that on-the-fly scans provide. Recent work supports the former claim by approximating a continuous measurement from a dense static scan through post-processing of the diffraction data. ${ }^{22} \mathrm{We}$ found that a line spacing comparable to the static scans gives good results if the spacing is significantly reduced along the continuous fast axis. The smaller step size along the continuous direction causes no significant increase in scan overhead. It produces better conditioned datasets by reducing the number of modes to be recovered while giving more diffraction patterns to make the solution more robust. It should be noted that because of the dead time between frames of only $10 \mu \mathrm{s}$, there is no significant scanning time penalty for segmenting the continuous acquisitions into more frames.

Although here we limited the continuous movement to the horizontal direction, there is no fundamental hurdle to devise a scan pattern that completely eliminates repositioning times of the piezos in 2D. Such acceleration constitutes a significant advance in the applicability of ptychography, making it easier to measure multiple samples in 2D and 3D or to measure large representative volumes for biology or materials science. We expect that combining these on-the-fly scans with fast algorithms that achieve sub-second reconstructions $^{23}$ will soon provide microscopists with a nearly real-time high-resolution imaging technique.

P.M.P. acknowledges financial support from an Erasmus Mundus Masters scholarship and the Paul Scherrer Institut. This work was supported in part by the European Research Council (FP7, StG 279753).

${ }^{1}$ J. M. Rodenburg and R. H. T. Bates, Philos. Trans. R. Soc., A 339, 521 (1992).

${ }^{2}$ P. Thibault, M. Dierolf, A. Menzel, O. Bunk, C. David, and F. Pfeiffer, Science 321, 379 (2008).

${ }^{3}$ M. Dierolf, A. Menzel, P. Thibault, P. Schneider, C. M. Kewish, R. Wepf, O. Bunk, and F. Pfeiffer, Nature 467, 436 (2010).

${ }^{4}$ B. Chen, M. Guizar-Sicairos, G. Xiong, L. Shemilt, A. Diaz, J. Nutter, N. Burdet, S. Huo, J. Mancuso, A. Monteith, F. Vergeer, A. Burgess, and I. Robinson, Sci. Rep. 3, 1177 (2013).

${ }^{5}$ P. Trtik, A. Diaz, M. Guizar-Sicairos, A. Menzel, and O. Bunk, Cem. Concr. Compos. 36, 71 (2013).

${ }^{6}$ B. Weinhausen, O. Saldanha, R. N. Wilke, C. Dammann, M. Priebe, M. Burghammer, M. Sprung, and S. Köster, Phys. Rev. Lett. 112, 088102 (2014).

${ }^{7}$ M. W. Jones, G. A. Van Riessen, B. Abbey, C. T. Putkunz, M. D. Junker, E. Balaur, D. J. Vine, I. McNulty, B. Chen, B. D. Arhatari, S. Frankland, K. A. Nugent, L. Tilley, and A. G. Peele, Sci. Rep. 3, 2288 (2013). 
${ }^{8}$ M. Guizar-Sicairos and J. R. Fienup, Opt. Express 16, 7264 (2008).

${ }^{9}$ A. Maiden, M. Humphry, M. Sarahan, B. Kraus, and J. Rodenburg, Ultramicroscopy 120, 64 (2012).

${ }^{10}$ M. Holler, A. Diaz, M. Guizar-Sicairos, P. Karvinen, E. Färm, E. Härkönen, M. Ritala, A. Diaz, J. Raabe, and O. Bunk, Sci. Rep. 4, 3857 (2014).

${ }^{11} \mathrm{P}$. Thibault and A. Menzel, Nature 494, 68 (2013).

${ }^{12}$ J. N. Clark, X. Huang, R. J. Harder, and I. K. Robinson, Phys. Rev. Lett. 112, 113901 (2014).

${ }^{13}$ E. Wolf, J. Opt. Soc. Am. 72, 343 (1982).

${ }^{14}$ E. Wolf, J. Opt. Soc. Am. 3, 76 (1986).

${ }^{15}$ I. Johnson, A. Bergamaschi, H. Billich, S. Cartier, R. Dinapoli, D. Greiffenberg, M. Guizar-Sicairos, B. Henrich, J. Jungmann, D. Mezza, A. Mozzanica, B. Schmitt, X. Shi, and G. Tinti, J. Instrum. 9, C05032 (2014).
${ }^{16}$ M. Holler, J. Raabe, A. Diaz, M. Guizar-Sicairos, C. Quitmann, A. Menzel, and O. Bunk, Rev. Sci. Instrum. 83, 073703 (2012).

${ }^{17}$ P. Thibault, M. Guizar-Sicairos, and A. Menzel, J. Synchrotron Radiat. 21, 1011 (2014).

${ }^{18} \mathrm{P}$. Thibault, M. Dierolf, O. Bunk, A. Menzel, and F. Pfeiffer, Ultramicroscopy 109, 338 (2009).

${ }^{19}$ P. Thibault and M. Guizar-Sicairos, New J. Phys. 14, 063004 (2012).

${ }^{20}$ M. V. Heel and M. Schatz, J. Struct. Biol. 151, 250 (2005).

${ }^{21}$ J. Vila-Comamala, A. Diaz, M. Guizar-Sicairos, A. Mantion, C. M. Kewish, A. Menzel, O. Bunk, and C. David, Opt. Express 19, 21333 (2011).

${ }^{22}$ J. N. Clark, X. Huang, R. J. Harder, and I. K. Robinson, Opt. Lett. 39, 6066 (2014)

${ }^{23}$ S. Marchesini, D. Shapiro, H. Krishnan, and H.-T. Wu, Sharp Camera Project, 2014. 